\title{
A scoping review of causal methods enabling predictions under hypothetical interventions
}

\author{
Lijing Lin ${ }^{1 *}$ D, Matthew Sperrin ${ }^{1}$, David A. Jenkins ${ }^{1,2}$, Glen P. Martin ${ }^{1}$ and Niels Peek ${ }^{1,2,3}$
}

\begin{abstract}
Background: The methods with which prediction models are usually developed mean that neither the parameters nor the predictions should be interpreted causally. For many applications, this is perfectly acceptable. However, when prediction models are used to support decision making, there is often a need for predicting outcomes under hypothetical interventions.
\end{abstract}

Aims: We aimed to identify published methods for developing and validating prediction models that enable risk estimation of outcomes under hypothetical interventions, utilizing causal inference. We aimed to identify the main methodological approaches, their underlying assumptions, targeted estimands, and potential pitfalls and challenges with using the method. Finally, we aimed to highlight unresolved methodological challenges.

Methods: We systematically reviewed literature published by December 2019, considering papers in the health domain that used causal considerations to enable prediction models to be used for predictions under hypothetical interventions. We included both methodologies proposed in statistical/machine learning literature and methodologies used in applied studies.

Results: We identified 4919 papers through database searches and a further 115 papers through manual searches. Of these, 87 papers were retained for full-text screening, of which 13 were selected for inclusion. We found papers from both the statistical and the machine learning literature. Most of the identified methods for causal inference from observational data were based on marginal structural models and g-estimation.

Conclusions: There exist two broad methodological approaches for allowing prediction under hypothetical intervention into clinical prediction models: (1) enriching prediction models derived from observational studies with estimated causal effects from clinical trials and meta-analyses and (2) estimating prediction models and causal effects directly from observational data. These methods require extending to dynamic treatment regimes, and consideration of multiple interventions to operationalise a clinical decision support system. Techniques for validating 'causal prediction models' are still in their infancy.

Keywords: Clinical prediction models, Statistical modeling, Causal inference, Counterfactual prediction

\footnotetext{
*Correspondence: lijing.lin@manchester.ac.uk

'Division of Informatics, Imaging and Data Science, Faculty of Biology,

Medicine and Health, University of Manchester, Manchester Academic Health

Science Centre, Manchester, UK

Full list of author information is available at the end of the article
}

(c) The Author(s). 2021 Open Access This article is licensed under a Creative Commons Attribution 4.0 International License, which permits use, sharing, adaptation, distribution and reproduction in any medium or format, as long as you give appropriate credit to the original author(s) and the source, provide a link to the Creative Commons licence, and indicate if changes were made. The images or other third party material in this article are included in the article's Creative Commons licence, unless indicated otherwise in a credit line to the material. If material is not included in the article's Creative Commons licence and your intended use is not permitted by statutory regulation or exceeds the permitted use, you will need to obtain permission directly from the copyright holder. To view a copy of this licence, visit http://creativecommons.org/licenses/by/4.0/. 


\section{Introduction}

Clinical prediction models (CPMs) aim to predict current diagnostic status or future outcomes in individuals, conditional on covariates [1]. In clinical practice, CPMs may inform patients and their treating physicians of the probability of a diagnosis or a future outcome, which is then used to support decision-making. For example, QRISK $[2,3]$ computes an individual's risk of developing cardiovascular disease within the next 10 years, based on their characteristics such as BMI, blood pressure, smoking status, and other risk factors. The $\mathrm{Na}$ tional Institute for Health and Care Excellence (NICE) guidelines indicate that anyone with an estimated QRISK above $10 \%$ should be considered for statin treatment [4]. These guidelines also state that initially, patients should be encouraged to implement lifestyle changes such as smoking cessation and weight loss. However, guidelines using such clinical prediction models can be problematic for two main reasons. First, there is often a lack of clarity concerning the estimand that a clinical prediction model is targeting [5]. To inform decision making about treatment initiation, one requires predicted risks assuming no treatment is given. This might be achieved by using a 'treatment-naïve' cohort (removing all patients who take treatment at baseline) $[2,3]$ or by incorporating treatment as a predictor variable in the model [6]. However, such approaches do not handle 'treatment drop-in': in which patients in the development cohort might start taking treatment post-baseline [7, 8]. One way to attempt to account for this is to censor patients at treatment initiation; however, this assumes that treatment initiation is not informative [9]. Second, these prediction models cannot indicate which of the potential treatment options or lifestyle changes would be best in terms of lowering an individual's future cardiovascular risk, nor can they quantify the future risk if that individual was given a treatment or lifestyle change $[10,11]$. With the lack of randomised treatment assignment such as in the observational studies, simply 'plugging in' the hypothetical treatment or intervention via the baseline covariates will rarely, if ever, give the correct hypothetical risks [11]. For example, there may be underadjustment due to residual confounding, or overadjustment of mediators or colliders.

To correctly aid such decision-making, one needs answers to 'what-if' questions. As an example, suppose we are interested in statin interventions for primary prevention of CVD and we would like to predict the 10-year risk of CVD with or without statin interventions at an individual level. The methods used to derive CPMs do not allow for the correct use of the model in answering such 'whatif questions, as they select and combine covariates to optimize predictive accuracy, not to predict the outcome distribution under hypothetical interventions [12, 13].
Nevertheless, end-users often mistakenly compare the contribution of individual covariates (in terms of risk predictions) and seek causal interpretation of model parameters [14]. Within a potential outcomes (counterfactual) framework, an emerging class of causal predictive models could enable 'what-if' queries to be addressed, specifically calculating the predicted risk under different hypothetical interventions. This enables targeted intervention, allows correct communication to patients and clinicians, and facilitates a preventative healthcare system.

There exists a vast literature on both predictive models and causal inference. While the use of prediction modelling to enrich causal inference is becoming widespread [15], the use of causal thinking to improve prediction modelling is less well studied [16]; however, its potential is acknowledged [13]. Our aim was therefore to identify methods for developing and validating 'causal prediction' models that use causal methods to enable risk estimation of outcomes under hypothetical interventions. We aimed to identify the main methodological approaches, their underlying assumptions, targeted estimands, and potential pitfalls and challenges with using the method. Finally, we aimed to highlight unresolved methodological challenges.

\section{Methods}

We aimed to identify all studies in which a form of causal reasoning is used to enable predictions for health outcomes under hypothetical interventions. To be clear, we were not interested in causal studies where the methods can solely be used to predict average or conditional causal effects [13].

Due to the available resources for reviewing large volumes of papers, the search was restricted to the health domain. We included both methodologies proposed in statistical/machine learning literature and methodologies used in applied studies. The review process adhered to Arksey and O'Malley's [17] scoping review framework and the preferred reporting items for systematic reviews and meta-analyses (PRISMA) statement [18]. We have also followed recommendations for conducting methodology scoping reviews as suggested in Martin et al. [19].

\section{Search strategy}

We systematically reviewed the literature available up to a cut-off date on December 31, 2019. The literature search was conducted in two electronic databases: Ovid MEDLINE and Ovid Embase, and searches were tailored to each database and restricted to English language publications. The search terms were designed by considering the intersection of prediction modelling and causal inference. Pre-existing search filters were utilised where possible such as those for prediction models [20]. Details of the search terms are included in the supplementary 
protocol. We were also aware, a priori, of several research groups that have published work on methods in related areas (listed in the supplementary protocol). We manually searched for any relevant recent publications within the past 4 years from these groups. In addition, we conducted backward citation search checking the references of identified papers, and a forward citation search using Google Scholar, which discovered papers referencing the identified papers.

\section{Selection of studies}

After the initial search, all titles and abstracts of papers identified by the search strategy were screened for eligibility by the lead author (LL). A random 3\% were screened by a second reviewer (DJ) to ensure reliability of the screening process. Any discrepancies between the reviewers were solved through mutual discussion, in consultation with a third reviewer, where needed (MS). The initial eligibility criteria, based on title and abstract screen, were as follows: (1) use causal reasoning in the context of health outcome prediction, specifically enabling prediction under hypothetical interventions; (2) describe original methodological research (e.g. peer reviewed methodological journal); or (3) applied research, which did not develop methodology, but state-of-the-art methodology was employed to address relevant causal prediction questions. We excluded studies that could only be used for causal effect estimation, and studies where standard clinical prediction models were used to infer conditional causal effects, e.g. [21]. However, we do not exclude papers that developed a novel method of allowing prediction under hypothetical intervention, even when the final goal was causal effect estimation. We excluded letters, commentaries, editorials, and conference abstracts with no information to allow assessment of proposed methods.

\section{Extraction}

Following the review aims, we extracted information from papers that were included after a full-text screening as follows:

1. Article type (summary/review, theoretical, modelling with application via simulation and/or observed data, purely applied paper);

2. Clinical topic area of analysis (e.g. CVD, HIV, cancer) for papers with application to observed data;

3. Intervention scenarios (single intervention vs multiple interventions); types of outcome and exposure examined (binary, time-to-event, count, continuous, other);
4. Information on targeted estimand and possible validation approaches for the proposed methods inferred by the review authors; stated possible sources of bias;

5. Stated assumptions; methodologies/methods used for the causal effect estimation and outcome prediction; main methodological novelty stated by the authors of identified papers;

6. Reported modelling strengths and limitations, and suggestions for the future work;

7. Availability of software/code.

The completed extraction table is available in the Supplementary File 1. Categorisation of papers was carried out during information extraction phase by synthesising the extracted information.

\section{Results}

Our database searches identified 4919 papers. We identified a further 115 papers through checking publications from known research groups, and forward and backward citation searching. Of these, 87 were retained for a fulltext screening, with 13 of these were deemed eligible for final inclusion, as listed in Table 1 . The process of study identification, screening, and inclusion is summarised in the PRISMA flowchart (Fig. 1).

The identified papers covered two main intervention scenarios: single intervention [22-27] and repeated interventions over time $[8,28-33]$ with nearly an equal amount of papers addressing average intervention effects (defined by a contrast of means of counterfactual outcomes averaged across the population) $[8,22,23,25,29$, 30] and conditional effects (defined as the contrast of covariate-specific means of the outcome under different intervention levels) [24, 26-28, 31-33]. Across the included papers, we identified two broad categories of methodological approaches for developing causal prediction models: (1) enriching prediction models with externally estimated causal effects, such as from metaanalyses of clinical trials and (2) estimating both the prediction model and causal effects from observational data. The majority of the identified papers (10 out of 13) fell into the latter category, which can be further divided according to intervention scenarios and included methods embedded within both statistical and machine learning frameworks. Table 1 describes part of the extracted information on each paper. The complete extraction table is available in the Supplementary File 1. In addition, we will illustrate the methods identified using the statin interventions for primary prevention of CVD example introduced previously, explaining each method and showing their differences in terms of targeted questions and corresponding estimand (Table 2). 
Table 1 Summary of included 13 papers (See Supplementary File 1 for the completed extraction table)

\begin{tabular}{|c|c|c|c|c|c|c|}
\hline Title & $\begin{array}{l}\text { Intervention } \\
\text { Scenario }\end{array}$ & $\begin{array}{l}\text { Clinical topic } \\
\text { area }\end{array}$ & $\begin{array}{l}\text { Types of } \\
\text { outcomes }\end{array}$ & Stated assumptions & Reported limitations & $\begin{array}{l}\text { Code } \\
\text { availability }\end{array}$ \\
\hline $\begin{array}{l}\text { Candido dos Reis, F. J. } \\
\text { et al. (2017) An updated } \\
\text { PREDICT breast cancer } \\
\text { prognostication and } \\
\text { treatment benefit } \\
\text { prediction model with } \\
\text { independent validation, }\end{array}$ & $\begin{array}{l}\text { Single } \\
\text { intervention, } \\
\text { Discrete choice, } \\
\text { Average effect }\end{array}$ & Breast cancer & Survival & $\begin{array}{l}\text { Generalisability of effect } \\
\text { from clinical trial. }\end{array}$ & $\begin{array}{l}\text { Prediction of non-breast } \\
\text { cancer deaths was excel- } \\
\text { lent in the model develop- } \\
\text { ment data set but could } \\
\text { under-predict or over- } \\
\text { predict in the validation } \\
\text { data sets. }\end{array}$ & $\begin{array}{l}\text { Stata code } \\
\text { are } \\
\text { available } \\
\text { from the } \\
\text { author on } \\
\text { request. }\end{array}$ \\
\hline
\end{tabular}

19(1), 58

Brunner, F. J. et al. (2019)

Application of non-HDL cholesterol for populationbased cardio-vascular risk stratification: results from the Multinational Cardiovascular Risk Consortium., The Lancet 394.10215: 2173-2183.

Silva, R. (2016),

Observational-

Interventional Priors for

Dose-Response Learning.

In Advances in Neural In-

formation Processing Systems 29.

Van Amsterdam, W. A. C. et al. (2019). Eliminating biasing signals in lung

cancer images for prognosis predictions with deep learning. npj Digital Medicine, 2(1), 1-6.

Alaa, A. M., \& Van Der

Schaar, M. (2017). Bayesian Inference of Individualized Treatment Effects using Multi-task Gaussian Processes. In Advances in Neural Information Processing Systems 30

Arjas, E. (2014) Time to Consider Time, and Time to Predict? Statistics in Biosciences. Springer New York LLC, 6(2), pp. 189-203

Pajouheshnia R. et al. (2020) Accounting for time-dependent treatment use when developing a prognostic model from observational data: A review 74(1).

Sperrin, M. et al. (2018) Using marginal structural models to adjust for treatment drop-in when developing clinical prediction models, Statistics in Medicine. John Wiley \& Sons, Ltd, 37(28), pp. 4142-
Single CVD Binary

intervention,

Discrete choice, Average effect

\section{Single}

intervention,

Treatment dose

(continuous),

Conditional

effect

Single

intervention,

Discrete choice

(0/1), Average

effect

Single

intervention

Discrete choice

$(0 / 1)$,

Conditional

effect

\section{Single}

intervention,

Discrete choice,

Conditional

effect

\section{Multiple} intervention

Discrete choice

$(0 / 1)$; Average

effect

Multiple

intervention;

Discrete choice

(0/1);

Conditional

effect

for

cardiovascular

patients

patients ear infections

\section{Chronic}

Infant Health Continuous A(1) and additionally: It is and

Development

Program (IHDP)

\section{Lung caner Survival}

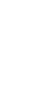

possible to collect interventional data such that treatments are controlled to contain important
The therapeutic benefit of lipid-lowering intervention investigated in the study is based on a hypothetical model that assumes a stable reduction of nonHDL cholesterol.

$A(1), A(2)$ and additionally: An image is hypothesized information for the clinical prediction task. The collider can be measured from the image.

\section{IHDP \& Heart Continuous/ A(2)}

transplantation Survival times

\section{Acute middle Survival $\quad A(2)$ and local} independence obstructive pulmonary disease (COPD)

$$
\text { Survival } \quad A(1) \text { and } A(2)
$$

Binary

$A(1)$ and $A(2)$
(1) Data limitation in the derivation cohort. (2) Strong clinical assumption that treatment effects are sustained over a much longer term than has been studied in clinical trials.

(1) Computation complexity. (2) Have not discussed at all the important issue of sample selection bias. (3) Generalisability issue.

(1) Provide an example of how deep learning and structural causal models can be combined. Methods combining machine learning with causal inference need to be further developed.

(1) No experiments regarding outcome prediction accuracy. (2) The computational burden is dominated by the $\mathrm{O}(\mathrm{n} 3)$ (matrix inversion on line 13 in Alg.1.

In studies involving real data the computational challenge can become formidable and even exceed what is feasible in practice.

A very strong indication for treatment will result in structural non-positivity leading to biased estimates of treatment-naïve risk.

(1) Have not modelled statistical interaction between treatment and prognostic factors; (2) Did not explicitly model statin discontinuation; (3) Only consider single treatment.
Reported using $\mathrm{R}$ but codes not available

Code available from OLS

Code available from OLS

Code available from authors' website. 
Table 1 Summary of included 13 papers (See Supplementary File 1 for the completed extraction table) (Continued)

\begin{tabular}{|c|c|c|c|c|c|c|}
\hline Title & $\begin{array}{l}\text { Intervention } \\
\text { Scenario }\end{array}$ & $\begin{array}{l}\text { Clinical topic } \\
\text { area }\end{array}$ & $\begin{array}{l}\text { Types of } \\
\text { outcomes }\end{array}$ & Stated assumptions & Reported limitations & $\begin{array}{l}\text { Code } \\
\text { availability }\end{array}$ \\
\hline \multicolumn{7}{|l|}{4154.} \\
\hline $\begin{array}{l}\text { Lim, B. et al. (2018). } \\
\text { Forecasting Treatment } \\
\text { Responses Over Time } \\
\text { Using Recurrent Marginal } \\
\text { Structural Networks. In } \\
\text { Conference on Neural } \\
\text { Information Processing } \\
\text { Systems } 32 \text {. }\end{array}$ & $\begin{array}{l}\text { Multiple } \\
\text { intervention; No } \\
\text { restriction on } \\
\text { treatment } \\
\text { choices; } \\
\text { Average effect }\end{array}$ & $\begin{array}{l}\text { Cancer growth } \\
\text { and treatment } \\
\text { responses }\end{array}$ & No restriction & $A(2)$ & NA & $\begin{array}{l}\text { Code } \\
\text { available } \\
\text { from OLS }\end{array}$ \\
\hline $\begin{array}{l}\text { Bica, I. et al. } \\
\text { (2020). Estimating } \\
\text { Counterfactual Treatment } \\
\text { Outcomes over Time } \\
\text { through Adversarially } \\
\text { Balanced Representations. } \\
\text { ICLR } 2020\end{array}$ & $\begin{array}{l}\text { Multiple } \\
\text { intervention; } \\
\text { Discrete } \\
\text { treatment } \\
\text { choices; } \\
\text { Average effect }\end{array}$ & $\begin{array}{l}\text { Treatment } \\
\text { response in a } \\
\text { tumour growth } \\
\text { model }\end{array}$ & No restriction & $A(2)$ & $\begin{array}{l}\text { Additional theoretical } \\
\text { understanding is needed } \\
\text { for performing model } \\
\text { selection in the causal } \\
\text { inference setting with } \\
\text { time-dependent treat- } \\
\text { ments and confounders. }\end{array}$ & $\begin{array}{l}\text { Code } \\
\text { available } \\
\text { from } \\
\text { authors' } \\
\text { website. }\end{array}$ \\
\hline $\begin{array}{l}\text { Xu, Y. et al. (2016) A } \\
\text { Bayesian Nonparametric } \\
\text { Approach for Estimating } \\
\text { Individualized Treatment- } \\
\text { Response Curves. Edited } \\
\text { by F.Doshi-Velez et al. } \\
\text { PMLR, pp. 282-300. }\end{array}$ & $\begin{array}{l}\text { Multiple } \\
\text { intervention; } \\
\text { Discrete } \\
\text { treatment } \\
\text { choices; } \\
\text { Conditional } \\
\text { treatment effect }\end{array}$ & $\begin{array}{l}\text { (1) kidney } \\
\text { function } \\
\text { deterioration in } \\
\text { ICU; (2) the } \\
\text { effects of } \\
\text { diuretics on } \\
\text { fluid balance. }\end{array}$ & Continuous & $A(2)$ & NA & NA \\
\hline $\begin{array}{l}\text { Soleimani, H. et al. (2017). } \\
\text { Treatment-response } \\
\text { models for counterfactual } \\
\text { reasoning with } \\
\text { continuous-time, } \\
\text { continuous-valued inter- } \\
\text { ventions. In Uncertainty in } \\
\text { Artificial Intelligence. Pro- } \\
\text { ceedings of the 33rd Con- } \\
\text { ference, UAl } 2017 \text {. }\end{array}$ & $\begin{array}{l}\text { Continuous- } \\
\text { time } \\
\text { intervention; } \\
\text { Continuous- } \\
\text { valued treat- } \\
\text { ments; Condi- } \\
\text { tional treatment } \\
\text { effect }\end{array}$ & $\begin{array}{l}\text { Modelling } \\
\text { physiologic } \\
\text { signals with } \\
\text { EHRs for } \\
\text { treatment } \\
\text { effects on renal } \\
\text { function }\end{array}$ & No restriction & $A(2), A(3)$ & $\begin{array}{l}\text { While this approach relies } \\
\text { on regularisation to } \\
\text { decompose the observed } \\
\text { data into shared and } \\
\text { signal-specific compo- } \\
\text { nents, new methods are } \\
\text { needed for constraining } \\
\text { the model in order to } \\
\text { guarantee posterior } \\
\text { consistency of the sub- } \\
\text { components of this model. }\end{array}$ & NA \\
\hline $\begin{array}{l}\text { Schulam, P., \& Saria, S. } \\
\text { (2017). Reliable Decision } \\
\text { Support using } \\
\text { Counterfactual Models. In } \\
\text { Advances in Neural } \\
\text { Information Processing } \\
\text { Systems } 30 .\end{array}$ & $\begin{array}{l}\text { Continuous- } \\
\text { time } \\
\text { intervention; } \\
\text { Continuous- } \\
\text { valued treat- } \\
\text { ments; Condi- } \\
\text { tional treatment } \\
\text { effect }\end{array}$ & $\begin{array}{l}\text { Applicable to } \\
\text { data from EHR } \\
\text { but not restrict } \\
\text { to such } \\
\text { medical } \\
\text { settings }\end{array}$ & $\begin{array}{l}\text { Continuous- } \\
\text { time; no } \\
\text { restriction on } \\
\text { data type }\end{array}$ & $A(2), A(3), A(4)$ & $\begin{array}{l}\text { (1) the validity of the CGP } \\
\text { is conditioned upon a set } \\
\text { of assumptions that are, in } \\
\text { general, not testable. The } \\
\text { reliability of approaches } \\
\text { therefore critically depends } \\
\text { on the plausibility of those } \\
\text { assumptions in light of } \\
\text { domain knowledge. }\end{array}$ & $\begin{array}{l}\text { Code } \\
\text { available } \\
\text { from } \\
\text { authors' } \\
\text { website. }\end{array}$ \\
\hline
\end{tabular}

Abbreviations for 'Stated Assumptions': A(1) Relevant directed acyclic graphs (DAGs) available, $A(2)$ Identifiability conditions (consistency, exchangeability, and positivity; or sequential version of consistency, exchangeability, and positivity for time-varying treatments), $A(3)$ Continuous-time exchangeability and $A(4)$ Noninformative measurement times. Other abbreviations: EHR electronic health record, $R C T$ randomised controlled trial

\section{Combining causal effects measured from external information}

Three papers [22-24] were identified as developing models with combined information from different sources to address single treatment effect. Candido dos Reis et al. [22] and Brunner et al. [23] took a two-stage approach, in which treatment effect estimates from external sources such as RCTs and meta-analyses were first identified, then combined with prediction models to allow predictions under treatment. In the statins for CVD example, the method proposed by Candido dos Reis et al. [22] corresponds to developing a CPM including individuals who take statin at baseline, where the coefficient for the statins variable in the model is fixed to the statin effects estimated from trials. Brunner et al. [23] developed a CPM for cardiovascular risk which was then combined with an externally estimated equation of proportional risk reduction per unit LDL cholesterol reduction to aid decision making in lipid-lowering treatment usage.

In addition to the above two-stage approach borrowing causal information estimated externally into predictive models, a one-stage approach, proposed by Silva [24], was also identified where the two sources of data, interventional and observational, were jointly modelled for causal prediction. This approach was applied in a 


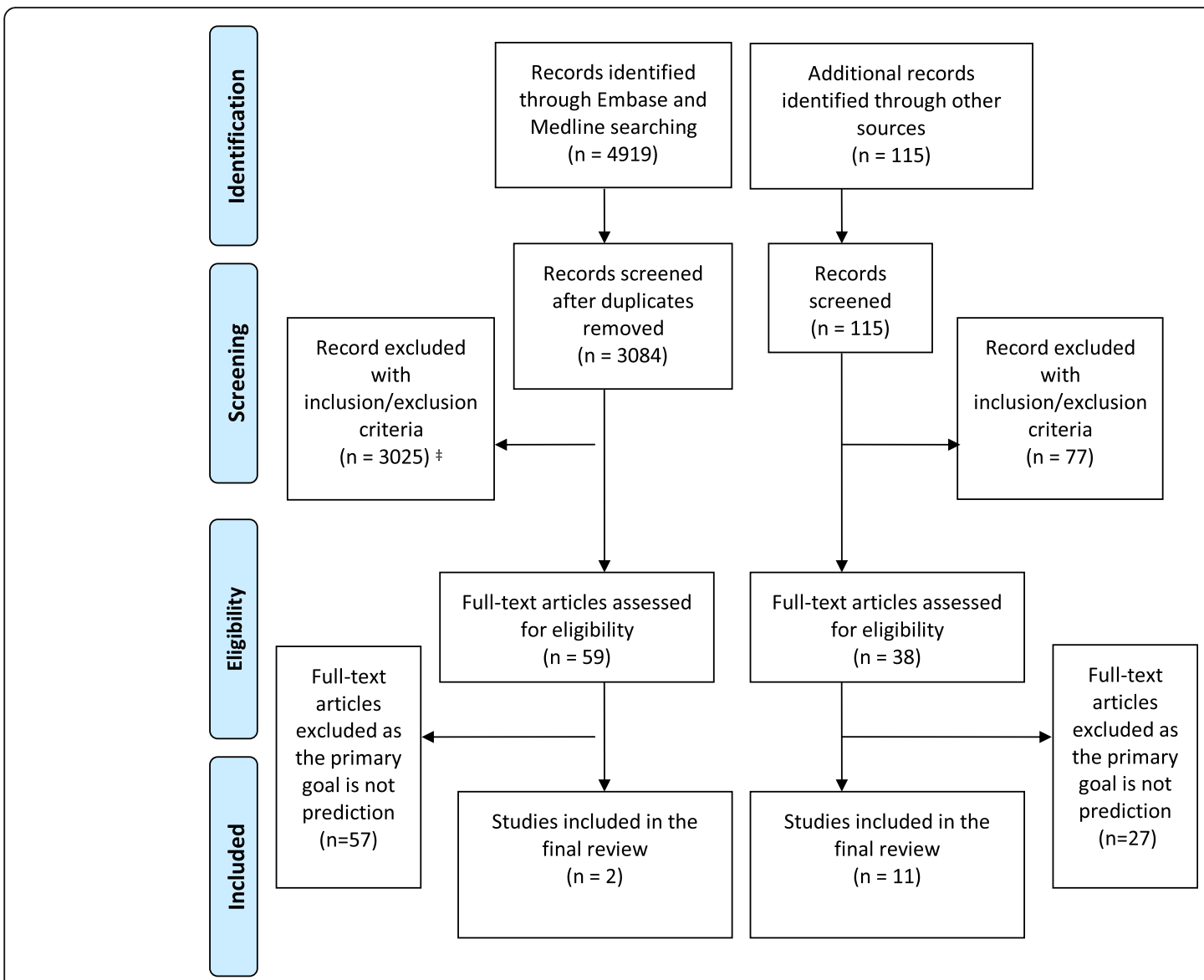

Fig. 1 PRISMA flow diagram. ${ }^{\ddagger}$ The majority of papers excluded at this stage did not meet our first inclusion criterion: that is, they did not focus on prediction under hypothetical interventions; these papers either did not use prediction models at all, or only used prediction modelling to enrich causal inference

scenario where it is possible to collect interventional data such that treatments were controlled but where sample sizes might be limited. The idea was to transform observational data into informed priors under a Bayesian framework to predict the unbiased dose-response curve under a pre-defined set of interventions, or 'dose'.

All the three approaches above are limited to a single intervention type and intervening at a single point in time, where, in a considered trial protocol, the intervention may follow-up for a certain length of time during which its choice is maintained (e.g. the initialisation of statin intervention). Approaches that directly apply the externally estimated causal effects into CPMs assume that the estimated causal effects are generalisable to the population in which one wishes to apply the prediction model. Equally, combining individual data from both sources (i.e. the one-stage approach) ignored the issue of sample selection bias, which was highlighted in [24]. Additionally, the one-stage approach can become computationally intensive as the size of the observational and number of treatment levels increase.

\section{Estimating both a prediction model and causal effects} from observational data

A total of 10 papers discussed modelling predictions under interventions entirely from observational data. Approaches from these papers can be further divided into two categories: (1) methods considering only one intervention at a single time point [25-27], as discussed in Section 3.2.1, and (2) methods allowing timedependent interventions [8, 28-33], as discussed in Section 3.2.2.

\section{Counterfactual prediction models that consider an intervention at a single point in time}

In our running example, this corresponds to a decision at a single time of whether to prescribe statins for CVD prevention. It does not account for whether statins are discontinued or started at any subsequent time.

Related to average treatment effect estimation Decision-making on whether to intervene on treatment requires an unbiased estimate of the treatment effect at 
Table 2 Illustration of methods in different categories using an example of statin intervention in primary prevention of CVD

\begin{tabular}{|c|c|c|c|}
\hline Approach cat & gories & Refs & Targeted estimand \\
\hline $\begin{array}{l}\text { Combining } \\
\text { causal effects } \\
\text { measured } \\
\text { from external } \\
\text { information }\end{array}$ & Two-stage approach & $\begin{array}{l}\text { Candido dos } \\
\text { Reis et al. [22] }\end{array}$ & $\begin{array}{l}\text { Risk of CVD under } \\
\text { intervention of taking or } \\
\text { not taking statin at } \\
\text { baseline (and, in a } \\
\text { considered trial protocol, } \\
\text { following-up for a certain } \\
\text { length of time during } \\
\text { which statin choice is } \\
\text { maintained): } E\left(Y^{\left(A_{0}\right)} \mid X_{0}\right)\end{array}$ \\
\hline
\end{tabular}

Potential pitfalls/ challenges

Efficacy/effectiveness gap when translating trial results to routine care. Comparability of trail and observed populations (selection bias).

Inflating the baseline cholesterol for individuals receiving statin by a certain level has assumed that 'statins had a moderate effect on lipid reduction and was initiated late during lifetime', and that statins operate only through cholesterol, i.e. ignores any other causal pathways.

One-stage approach

Silva [24]

Risk of CVD under intervention of taking statin of dosage $a_{i,}(i=1$, $\left(X_{0}\right)$.

Sample selection bias between the interventional data and observationa data.

prediction

model and

causal effects

from

observational

data
Van

Amsterdam

et al. [25]

\begin{tabular}{|c|c|c|c|}
\hline $\begin{array}{l}\text { Single } \\
\text { intervention }\end{array}$ & $\begin{array}{l}\text { Related to } \\
\text { average } \\
\text { treatment } \\
\text { effect } \\
\text { estimation }\end{array}$ & $\begin{array}{l}\text { Van } \\
\text { Amsterdam } \\
\text { et al. [25] }\end{array}$ & $\begin{array}{l}\text { Risk of CVD under } \\
\text { intervention of taking/not } \\
\text { taking statin at baseline, } \\
\text { regardless of future: } E\left(Y^{\left(A_{0}\right)}\right. \\
\left(X_{0}\right) \text {. }\end{array}$ \\
\hline
\end{tabular}

estimation $\left.\quad \mid X_{0}\right)$

Related to conditional treatment effect estimation
Alaa et al. [26]

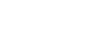

Risk of CVD under taking statin at baseline, regardless of future: $E\left(Y^{\left(A_{0}\right)} \mid X_{0}\right)$. intervention of taking/not
An over-simplified causal structure can lead to biased estimates of causal effects, e.g. when there exists more than one collider that were not observed but whose information were contained in the prognostic factors.

Without careful examination of causal structure within the variables, biased association between treatment and outcome can be introduced.
Arjas [27]
Risk of CVD under intervention of taking/not taking statin at baseline, regardless of future: $E\left(Y^{\left(A_{0}\right)} \mid \bar{H}_{0}\right)$.
Potentially biased estimate due to misspecification of intensity functions required in the outcome hazard model.
Timedependent treatments
MSMs within a Pajouheshnia prediction model et al. [8]
Risk of CVD under interventions of taking/not taking statin at baseline
The effectiveness of bias correction depends on a correct specification of

\section{Exemplary methods/} evaluations

Develop a CPM using individuals who take statin at baseline with the coefficient for treatment variable in the model fixed to the statin effects estimated from trials.

Inflate the baseline cholesterol of individuals receiving statin (by 30\% e.g.). Develop a CPM using all individuals. Combine the predicted individual-level CVD risk with an effect equation estimated from trials to get the absolute risk under intervention.

Individual patient data from RCTs and observational clinical data are combined under a Bayesian framework to predict risk under intervention. Use MCMC to approximate the posterior distributions of the parameters in the model.

Use a CNN to separate the unobserved collider information from other risk factors while using the last layer resembling linear regression to include the treatment variable as a covariate for risk prediction under intervention.

Estimate the outcome curves for the treated samples and untreated samples simultaneously using the signal-in-whitenoise model. The estimation of model is done through one loss function, known as the precision in estimating heterogeneous effects (PEHE).

Use treatment history and other risk factors measured over-time to set up a Bayesian model to estimate the outcome risk intensity function over time. For prediction, given an individual's measurements up to time $t$, estimate the risk under a single intervention by applying MCMC on the predictive distributions.

Assume a causal structure. Estimate treatment censoring probabilities by 
Table 2 Illustration of methods in different categories using an example of statin intervention in primary prevention of CVD (Continued)

\begin{tabular}{|c|c|c|c|}
\hline Approach categories & & Refs & Targeted estimand \\
\hline \multirow[t]{3}{*}{$\begin{array}{l}\text { and } \\
\text { treatment- } \\
\text { confounder } \\
\text { feedback }\end{array}$} & framework & & $\begin{array}{l}\text { and/or some other times } \\
\text { at the future: } E( \\
\left.Y(\bar{A}(0, K)=0) \mid X_{0}\right)\end{array}$ \\
\hline & & $\begin{array}{l}\text { Sperrin et al. } \\
\text { [28] }\end{array}$ & $\begin{array}{l}\text { Risk of CVD under } \\
\text { interventions of taking/not } \\
\text { taking statin at baseline } \\
\text { and/or some other times } \\
\text { at the future: } E( \\
\left.Y(\bar{A}(0, K)=0) \mid X_{0}\right)\end{array}$ \\
\hline & & Lim et al. [29] & $\begin{array}{l}\text { Risk of CVD and/or other } \\
\text { outcomes of interest (e.g. } \\
\text { cholesterol, SBP, etc) under } \\
\text { multiple interventions } \\
\text { planned for the next } \tau \\
\text { timesteps from current } \\
\text { time, given an observed } \\
\text { history } \bar{H}_{0} \text { : } \\
E\left(Y_{T}^{(\bar{A}(0, \tau-1))} \mid \bar{H}_{0}\right) .\end{array}$ \\
\hline
\end{tabular}

Methods based on balanced representation approach
Bica et al. [30]

(1)

The effectiveness of bias correction depends on a correct specification of treatment model. Requires agreement between the prediction model and the set of variables required for conditional exchangeability.

Requires agreement between the prediction model and the set of variables required for conditional exchangeability.

Potential confounders as no careful examination of causal structure.
Potential bias due to

strong assumptions on g-computation for correcting time-varying confounding

Xu et al. [31]

Cholesterol or other continuous outcome of interest (univariate) at any time $t$ in the future, under a sequence of model structure and possible model misspecification. interventions planned irregularly from current time till $t, \bar{A}_{0}<t$, given observed history: $E\left(Y_{t}^{\left(\bar{A}_{0,<t}\right)} \mid\right.$ $\bar{H}_{0}$ ).

Soleimani et al. [32]
$E\left(Y_{t}^{\left(\bar{A}_{0,<t}\right)} \mid \bar{H}_{0}\right)$ : same as in the $X u$ et al. method except that now the outcome $Y$ can be multivariate (e.g.

\section{Exemplary methods/ evaluations}

fitting logistic regression
models in each of the follow-up periods and derive time-varying censoring weights. After censoring, develop the prognostic model using a weighted Cox model.

Assume a causal structure. Collect the baseline prognostic factors, treatments, and treatment confounders at each time point post-baseline. Compute IPTWs using a treatment model; with derived IPTWs, build a logistic regression for outcome prediction under treatments.

With observed treatment, covariate and outcome histories (allowing for multiple treatment options of different forms), develop a propensity network to compute the IPTW and a sequence-to-sequence model that predict the outcome under a planned sequence of interventions.

Build a counterfactual recurrent network to predict outcomes under interventions:

1. For the encoder network, use an RNN, with LSTM unit to build treatment invariant representations of the patient history $\Phi\left(\bar{H}_{t}\right)$ and to predict one-stepahead outcomes $Y_{t+1}$; 2. For the decoder network, use $\Phi\left(\bar{H}_{t}\right)$ to initialize the state of an RNN that predicts the counterfactual outcomes for future treatments.

With observed treatment/ covariate/outcome histories, estimate treatment-response trajectories using a Bayesian nonparametric or semiparametric approach: 1. Specify models for different components in the generalised mix-effect model for outcome prediction.

These usually include: treatment response, baseline regression (fixed effects), and random effects. For the case where 
Table 2 Illustration of methods in different categories using an example of statin intervention in primary prevention of CVD (Continued)

\begin{tabular}{|c|c|c|c|c|}
\hline Approach categories & Refs & Targeted estimand & $\begin{array}{l}\text { Potential pitfalls/ } \\
\text { challenges }\end{array}$ & $\begin{array}{l}\text { Exemplary methods/ } \\
\text { evaluations }\end{array}$ \\
\hline & & $\begin{array}{l}\text { simultaneously predict risk } \\
\text { of CVD, cholesterol and } \\
\text { SBP) and the treatment can } \\
\text { be both discrete-time and } \\
\text { continuous-time. }\end{array}$ & & $\begin{array}{l}\text { the treatments are } \\
\text { continuously-administrated, } \\
\text { model the treatment re- } \\
\text { sponse using LTI dynamic } \\
\text { systems (Soleimani et al). } \\
\text { 2. Choose priors for these } \\
\text { models based on expert } \\
\text { domain knowledge. } \\
\text { 3. Use maximum a } \\
\text { posteriori (MAP) (Soleimani } \\
\text { et al.) or MCMC (Xu et al.) } \\
\text { to approximate the } \\
\text { posterior distributions of } \\
\text { the parameters in the } \\
\text { proposed model. }\end{array}$ \\
\hline & $\begin{array}{l}\text { Schulam } \\
\text { et al. [33] }\end{array}$ & $\begin{array}{l}E\left(Y_{t}^{\left(\bar{A}_{0, k t}\right)} \mid \bar{Y}_{0}, \bar{A}_{<0}\right) \text { : same as } \\
\text { in the Soleimani et al. } \\
\text { method except that the } \\
\text { observations only include } \\
\text { intervention and outcome } \\
\text { histories. }\end{array}$ & $\begin{array}{l}\text { Potential bias due to } \\
\text { strong assumptions on } \\
\text { model structure and } \\
\text { possible model } \\
\text { misspecification. Lack of } \\
\text { effect heterogeneity due } \\
\text { to omitting baseline } \\
\text { covariates. }\end{array}$ & $\begin{array}{l}\text { With observed histories, } \\
\text { jointly model intervention } \\
\text { and outcomes using a } \\
\text { marked point process } \\
\text { (MPP): } \\
\text { 1. Specify models for the } \\
\text { components in the MPP } \\
\text { intensity function: event } \\
\text { model, outcome model, } \\
\text { action (intervention) model. } \\
\text { The parameterization of the } \\
\text { event and action models } \\
\text { can be chosen to reflect } \\
\text { domain knowledge. The } \\
\text { outcome model is } \\
\text { parameterized using a GP. } \\
\text { 2. Maximise the likelihood } \\
\text { of observational traces over } \\
\text { a fixed interval to estimate } \\
\text { the parameters. }\end{array}$ \\
\hline
\end{tabular}

baseline. Assuming that the Directed Acyclic Graph (DAG) that encodes the relationship between all the relevant variables is known, then do-calculus [34] provides an indication of whether this can be achieved in the setting of observational data with the required causal assumptions. For example, including a collider in the model (a variable caused by both treatment and outcome) will lead to biased estimates of treatment effects on the outcome. A more complex scenario appears when the collider itself cannot be directly observed, but its information is contained in other prognostic factors. Van Amsterdam et al. [25] proposed a deep learning framework to address this particular scenario. Their goal is to predict survival of lung cancer patients using CT-scan images, in which case factors such as tumor size and heterogeneity are colliders that cannot be directly observed but can be measured from the image. The authors proposed a multi-task prediction scheme embedded in a convolutional neural network (CNN) framework (a non-linear model often used with images) which can simultaneously estimate the outcome and the collider. It used a $\mathrm{CNN}$ to separate the unobserved collider information from images while enabling the treatment to be appropriately included as a covariate for risk prediction under intervention. As there is no modelling of interactions between treatment and other covariates, this approach only addresses average treatment effects.

Van Amsterdam et al. [25] have demonstrated that deep learning can in principal be combined with insights from causal inference to estimate unbiased treatment effect for prediction. However, the causal structure applied therein was in its simplest form, and further developments are needed for more realistic clinical scenarios where, e.g. there is confounding for treatment assignment, or a treatment effect modifier exists within the image.

Related to conditional treatment effect estimation Let $Y^{(a)}$ denote the potential outcome under an intervention $a$, for example one's risk of CVD or cholesterol level under intervention of taking statin. Conditional treatment effects for subjects with a covariate $X=x$ in a population at a single time point is defined as $T(x)=$ $E\left[Y^{(1)}-Y^{(0)} \mid X=x\right]$, and our goal here is to estimate the 
counterfactual prediction of $E\left[Y^{(a)} \mid X=x\right], a \in\{0,1\}$. In an RCT, given complete randomisation-i.e., $a$ is independent of $Y^{(a)}$ and $X$, under consistency, one can estimate $E\left[Y^{(a)} \mid X=x\right]$ by fitting a prediction model to the treated arm $(a=1)$ and the control arm $(a=0)$, respectively. The technique is often used in estimating conditional treatment effects [21,35] or identifying subgroups from RCTs [36, 37], whereas our focus is counterfactual prediction under interventions. In Alaa et al. [26], under a set of assumptions, this technique was adapted for counterfactual prediction with observational data, which used a more complex regression model to address for selection bias in the observational dataset.

Alaa et al. [26] adopted standard assumptions of unconfoundedness (or ignorability) and overlap (or positivity), which is known as the 'potential outcomes model with unconfoundedness'. Their idea is to use the signal-inwhite-noise model for the potential outcomes and estimate two target functions, the treated and the untreated, simultaneously with training data. The estimation is done through one loss function, known as the precision in estimating heterogeneous effects (PEHE), which jointly minimises the error of factual outcomes and the posterior counterfactual variance, in such a way to adjust for the bias between the treated and untreated groups. The counterfactual prediction for either treated or untreated can then be made through the estimated posterior mean of two potential outcome functions. Since the ground truth counterfactual outcomes are never available in real-world observational datasets, it is not straightforward to evaluate causal prediction algorithms and compare their performances. A semi-synthetic experimental setup was adopted in [26], where covariates and treatment assignments are real but outcomes are simulated.

For the longitudinal setting where the event history is fully observed, Arjas [27] adopted a marked point process (MPP) framework with a Bayesian non-parametric hazard model to predict the outcome under a single intervention. Point processes are distributions over sequences of time points, and a marked point process is made by attaching a characteristic (a Mark) to each point of the process [38]. The idea is to incorporate all observed events in the data, including past treatments, covariates, and outcome of interest, into a single MPP: $\left\{\left(T_{n}, X_{n}\right): n \geq 0\right\}$, where $T_{0} \leq T_{1} \leq \cdots$ are the ordered event times and $X_{n}$ is a description of the event occurring at $T_{n}$. The model assumed local independence-i.e. the intensities of events (that is, the probability of an event occurring in an infinitesimal time interval) when considered relative to the histories $\bar{H}_{t}$ are locally independent of outcome risk functions in the model. Under this assumption, in order to define a statistical model for MPP, it suffices to specify the outcome intensities with respect to $\bar{H}_{t}$ and there is no need for other event time intensities. Prediction under hypothetical interventions can be then made by evaluating the corresponding predictive probabilities in the Bayesian posterior predictive setting given the data.

Both methods in this subsection can be computationally intensive as the number of observed samples increased. This could be ameliorated using conventional sparse approximations [26, 39]. Both methods are limited to binary interventions, and prediction via treatment effect estimation can only make counterfactual prediction for outcomes with or without intervention.

\section{Counterfactual prediction models that consider time- dependent treatments and treatment-confounder feedback} Papers included in this category $[8,28-33]$ covered three types of approaches to deal with scenarios where the treatments of interest and confounders vary over time. One example of such confounding is in the sequential treatment assignment setting, where doctors use a set of variable measurements, at the current time or in the past, to determine whether or not to treat, which in turn affects values of these variables at a subsequent time. For example, whether or not statins are taken at a particular time will affect cholesterol, and these subsequent cholesterol levels affect subsequent decisions about statins. The benefit of such approaches is that they allow consideration of a longer term treatment plan, such as comparing taking statins continuously for 10 years from baseline, versus not taking statins for the next 10 years. The assumptions needed for identifying unbiased treatment effects in such scenarios are consistency, positivity, and sequential ignorability.

Marginal structural models (MSMs) within a prediction model framework Consider in our running example the hypothetical risk of not taking statins for the next 10 years. To account for treatment drop-ins, i.e. treatments initiated post-baseline, one straightforward way is to censor patients at treatment initiation; however, this assumes that treatment initiation is noninformative about the baseline and time-dependent covariates. Pajouheshnia et al. [8] proposed censoring followed by reweighting using inverse probability of censoring weights (IPCW) to solve the issue of informative censoring in estimating treatment-naïve risk. The proposed method derived time-varying censoring weights by estimating the conditional probabilities of treatment initiation and then developed a weighted Cox model in the treatment-naïve pseudo-population. A more flexible way of addressing treatment drop-ins for hypothetical risk prediction is to use MSMs with inverse probability treatment weighting (IPTW), where a pseudo-population is created such that treatment selection will be unconfounded. Sperrin et al. [28] proposed combining MSM with predictive modelling approaches to adjust for 
confounding and generate prediction models that could appropriately estimate risk under the required treatment regimens. Following the classic development of IPTW for an MSM, the proposed methods develop two prediction models: a treatment model for computing the probability of receiving post-baseline treatments, and an outcome prediction model fitted with the derived weights and with these post-baseline treatments as well as terms for any interactions between treatment and other predictors included as predictors. By carefully defining the required estimand for the target prediction, the proposed framework could estimate risks under a variety of treatment regimens. In the statin example, this means that one can compare CVD risk under a range of different statin treatment plan, although the focus in the paper was on the 'never takes statins' hypothetical prediction. As with approaches described so far in this category, the model only considered a binary treatment (e.g. statins yes/no). The extension to multiple treatment choices for the proposed method is possible in principal, although the underlying causal structure and resulted model may become too complex.

Similarly to [28], Lim et al. [29] adopted the MSM combined with IPTW approach. Instead of using linear or logistic regression models, they embedded the concept into a deep learning framework and proposed a $R e-$ current Marginal Structural Network (RMSN). The model consisted of (1) a set propensity networks to compute treatment probabilities used for IPTW and (2) a prediction network used to determine the treatment response for a given set of planned interventions.

The benefit of RMSN is that, it can be configured to have multiple treatment choices and outcomes of different forms (e.g. continuous or discrete) using multiinput/multi-output RNNs. This means, in the statin example, one could consider different doses, and indeed consider alternative treatments as well. Treatment sequences can also be evaluated, and no restrictions were imposed on the prediction horizon or number of planned interventions. The use of LSTMs in computing the probabilities required for propensity weighting can also alleviate susceptibility of IPTWs to model misspecification. A drawback is that one needs a rich source of longitudinal data to train the model. Moreover, as in general in deep learning models, they lack a clear interpretation.

Methods based on balanced representation approach Matching approaches such as MSM or RMSN combined with IPTW above adjust for bias in the treatment assignments by creating a pseudo-population where the probability of treatment assignments does not depend on the timevarying confounders. Balanced representation approach, as proposed by Bica et al. [30], instead aimed for a representation $\Phi$ of the patient history $\bar{H}_{t}=\left(\bar{A}_{t-1}, \bar{X}_{t}\right)$ that was not predictive of treatment assignments. That is, in the case of two treatment assignments at time $t, P\left(\Phi\left(\bar{H}_{t}\right) \mid A_{t}\right.$ $=0)=P\left(\Phi\left(\bar{H}_{t}\right) \mid A_{t}=1\right)$. It can be shown that, in this way, estimation of counterfactual treatment outcomes is unbiased [40]. Bica et al. [30] proposed a counterfactual recurrent network (CRN) to achieve balancing representation and estimate unbiased counterfactual outcomes under a planned sequence of treatments (such as statins). CRN improved the closely related RMSN model proposed by Lim et al. [29] in a way that overcame the fundamental problem with IPTW, such as the high variance of the weights. As with RMSN, both models required hyperparameter tuning. As the counterfactual outcomes were never observed, hyperparameters in both models were optimised based on the error on the factual outcomes in the validation dataset. As noted by the authors in [30], more work on providing theoretical guarantees for the error on the counterfactuals is required.

Methods with g-computation for correcting timevarying confounding Three papers [31-33] were identified using g-computation to correct time-varying confounding and predicting treatment response curves under the potential outcome framework.

$\mathrm{Xu}$ et al. [31] developed a Bayesian non-parametric model for estimating conditional treatment response curves under the g-computation formula and provided posterior inference over the continuous response curves. In the statin example, this means that one can estimate cholesterol or any other continuous outcome of interest under a planned sequence of statin treatments (yes/no). The proposed method modelled the potential outcome using a generalized mixed-effects model combining the baseline progression (with no treatment prescribed), the treatment responses overtime, and noise. The goal was to obtain posterior inference for the treatment response and predict the potential outcomes given any sequence of treatments conditioned upon past treatments and covariate history. There are two limitations to the model here: (1) it assumes independent baseline progression and treatment response components, and (2) treatment response models rely on the additive treatment effects assumption and a careful choice of priors based on clinical details to be decided by domain experts.

Soleimani et al. [32] extended the approach in $\mathrm{Xu}$ et al. [31] in two ways: (1) to continuous-time setting with continuous-valued treatments and (2) to multivariate outcomes. This means, in the statin example, one could simultaneously predict e.g. risk of CVD, cholesterol, and systolic blood pressure (SBP) under a range of different statin treatment plans (allowing for different doses assigned at different time points). The model has its ability to capture the dynamic response after the 
treatment is initiated or discontinued by using linear time-invariant systems. Despite being a more flexible model than [31], this model did not overcome two limitations mentioned above.

Schulam and Saria [33] considered another continuoustime setting where both type and timing of actions may be dependent on the preceding outcome. In the statin example, this means both the statin dose and treatment time (initialisation or discontinuation) depend on the preceding cholesterol level. Here, one needs to predict how a continuous-time trajectory will progress under sequences of actions. The goal was to model action-outcome traces $D \equiv\left\{t_{i j}, Y_{i j}, a_{i j}\right\}_{i, j}$ : for each individual $i$ and irregularly sampled sequences of actions and outcomes. Schulam and Saria [33] proposed a counterfactual Gaussian process (CGP) model to model the trajectory and derived an adjusted maximum likelihood objective that learned the CGP from observational traces. The objective was derived by jointly modeling observed actions and outcomes using a marked point process (MPP). The potential outcome query can therefore be answered with the posterior predictive trajectory of the outcome model. A key limitation in this model is that it could not model heterogeneous treatment effects arising from baseline variables.

Counterfactual prediction models in this section using g-formula to correct for time-varying confounding are highly flexible and can be adopted for a variety of clinical settings. However, these methods rely on a set of strong assumptions in both discrete-time and continuous-time settings that are generally not testable; for the latter, Schulam and Saria [33] extended Robin's Sequential No Unobserved Confounders assumption to continuous-time case and also assumed Non-informative Measurement Times.

\section{Discussion}

In this study, we conducted a methodology scoping review, which has identified two main types of causal predictive modelling (methods that allow for prediction under hypothetical interventions), with the main differences between the methods being the source of data from which the causal effects are estimated. We identified that when the causal effects required for the predictions were fully estimated from the observational data, methods are available for predictions under interventions either at a single time point or varying over time. We have collated current approaches within this field and highlighted their advantages and limitations in the review.

There are recent studies that have performed a review of methods for causal inference all with different focuses: methods in the analyses of RCTs [41], methods based on graphical models [42] or DAGs [43], and methods targeting time-varying confounding [44]. Our work differs from these reviews, and, to our knowledge, is the first review to focus on methods enabling predictions under interventions (i.e. counterfactual prediction models). A recent review focused on how time-dependent treatment use should be handled when developing prediction models [8]. This clarified the targeted estimand of the clinical prediction model of interest and consider hypothetical risks under no interventions.

Our search terms, defined from the intersection of prediction modelling filters and causal inference keywords, have been made purposely broad to capture relevant literature, albeit with a high number of falsepositives driven by the heterogeneity in language across the fields. This could imply a challenge in devising a potentially more effective search strategy for identifying methodological papers on both fields, a challenge as highlighted in Martin et al. [19].

This review has synthesised a range of methods, embedded within both statistical and machine learning frameworks. These methods rely on the availability of the DAG that encodes the relationship between all the relevant variables, and a series of assumptions that make it possible to estimate counterfactual predictions from observational data. Approaches described here cover a wide range of data settings and clinical scenarios. Careful thoughts are needed before adopting these methods, and further challenges and gaps for future research remain, which we will discuss here.

Methods combining information from different sources, such as RCTs combined with observational data, provide a natural way to enable counterfactual predictions; however, challenges remain when combining these two settings. Their objectives are not necessarily complementary, leading to distinct populations included in each study (of possibly very different sample sizes), different sets of covariates being measured, and some potential measurement bias. Therefore, combining observational study with RCTs would need more careful consideration, and a good global guidance may be required. Harrell and Lazzeroni [45] laid out some initial steps one can follow toward an optimal decision making using both RCTs and electronic health record (EHR) data. We also refer the reader to the recent PATH (Predictive Approaches to Treatment effect Heterogeneity) Statement [46, 47], developed to provide guidance for predictive analyses of heterogeneity of treatment effects (HTE) in clinical trials. Predictive HTE analysis aims to express treatment effects in terms of predicted risks and predict which of 2 or more treatments will be better for a particular individual, which aligns closely with our review aim here. However, as motivated by the limitations in the conventional subgroup analyses in RCTs, predictive HTE analysis has focused on regression-based prediction in randomised trials for treatment effect estimation and subgroup identification. Such techniques 
can be adapted for the purpose of counterfactual prediction. For example, the predictive modelling used in estimating individualised causal effect in [21,35] was applied for counterfactual prediction in the included paper [30]. However, as the primary goal in predictive HTE analyses such as [21, 35] is not predicting the counterfactual outcome, we did not include them in our review, which may also be deemed as a limitation of this study.

Another obstacle in combining RCTs with observational study is that, while the estimand for causal inference is clearly defined, the prediction estimand, termed the predictimand by Van Geloven et al. [5], is often unclear in prediction models. There is an emergence of studies arguing that clearly defining the estimand in prediction is important $[28,43]$. Despite these challenges, and that relatively little work has been done in combining RCTs with patient observational data, it remains an opportunity to explore the interplay of these two areas, as noted in the recent survey by Bica et al. [48].

Several key challenges arise in dealing with multiple interventions. The term 'multiple treatments' has been commonly used throughout literature, especially when addressing time-varying treatments. However, the same term may refer to very distinct scenarios in different studies, and greater clarity is necessary. The first and the most often seen scenario is where multiple values/options are observed for a treatment variable, either at a single time point or over time. Treatments in this setting are indeed 'multivariate treatments'. Many approaches in this review are designated to deal with multivariate treatments $[24,30-33]$ or can in principal be extended to this case [25], [28]. However, except for the approach in [24], all methods assume treatment effects from different options to be independent; in [24], interactions between treatment options are modelled through the covariance matrix in the Gaussian process prior. Further methodological development could explore ways to incorporate treatment-treatment interactions into the model.

A second scenario of 'multiple treatments' is where there are interventions on several risk factors, which is substantially more complex, but also more realistic. For example, in clinical settings, one could intervene on different risk factors to prevent CVD, and possible interventions include giving antihypertensive drug or lipidlowering treatment, lifestyle changing (physical activity, smoking, and alcohol drinking), or a combination of them. As these interventions take effect on different parts of the causal structure for the outcome, changes in one factor may affect others, e.g., weight gain after smoking cessation [49]. Moreover, each clinical intervention scenario will require its own model for identifying treatment effects from observational data [11]. Recent studies on estimating causal effect under multiple interventions have explored methods such as marginal structural cox models [50] and parametric g-formula [51]. However, despite its apparent need in clinical practice as in the abovementioned example, there appears to be a lack of models for counterfactual predictions under multiple interventions, and future methodological development is required.

Treatment scenarios addressed so far in this review, both time-fixed and time-varying, are static interventions, i.e. treatment assignment under intervention does not depend on the post baseline covariates. In contrast to the static intervention is the dynamic treatment strat$e g y$, a rule in which treatments are assigned dynamically as a function of previous treatment and covariate history. Methods such as dynamic MSMs introduced by Orellana et al. [52] and independently by Van der Laan and Petersen [53], and variants of structural nested models (SNMs) introduced by Robins [54] were proposed to use observational data to estimate the optimal dynamic treatment regime. Embedding these methods within clinical prediction framework could enable counterfactual predicting under dynamic treatment allocation and support decision-making on optimal treatment rules, which presents a promising avenue for future research [55].

The most pressing problem to address for predictions under hypothetical interventions is model validation. Validation is a crucial step in prediction modelling (counterfactual or otherwise), but is challenging in the counterfactual space since that the counterfactual outcomes are not observable in the validation dataset. The included papers have by-passed this issue by noting that, models are fitted based on the error on the factual outcomes in the validation dataset. In this context, handling of treatment in validation of clinical prediction models has received some attention [56]. Pajouheshnia et al. [56] addressed the specific case of validating a prognostic model for treatment-free risk predictions in a validation set where risk-lowering treatments are used. However, further studies are required to extend the potential methods to address the more complex issue in validating counterfactual predictions, such as nondiscrete treatment types and non-parametric models as included in this review. While there is emerging research on developing a model validation procedure to estimate the performance of methods for causal effects estimation [57] and sensitivity analysis in causal inference [58], techniques are required to validate the models tailored for counterfactual prediction. Just as domain knowledge is important in causal inference before real-world deployment, it is also important in validating counterfactual prediction, and integrating data generated from RCTs and observational studies and their corresponding models provides a promising way to aid the process [48]. 


\section{Conclusions}

Prediction under hypothetical intervention is an emerging topic, with most methodological contributions published after 2015. This is now an active area of research in both the statistics and machine learning communities. Available methods for causal predictive modelling can be divided into two approaches. The first combines data from randomised controlled trials with observational data, while the second approach uses observational data only. We recommend using causal effects from randomised controlled trials where possible, combining these with prediction models estimated from observational data, as this alleviates the required assumptions for the causal contrasts to be unbiased. However, further theoretical guarantees are required regarding triangulating data from multiple sources. As well as the data sources available, the targeted estimand needs careful thought, and a relevant approach for the required estimand should be chosen. For example, marginal structural models can be used if observational data are used to make hypothetical predictions concerning an intervention that is sustained into the future. However, techniques to validate such models, and approaches for hypothetical risks under multiple or dynamic intervention scenarios, are under-investigated.

\section{Supplementary Information}

The online version contains supplementary material available at https://doi. org/10.1186/s41512-021-00092-9.

\section{Additional file 1}

Additional file 2

\section{Acknowledgments}

We thank two reviewers for their thoughtful comments and suggestions, which helped improve and clarify this manuscrip, and have undoubtedly strengthened the final version.

\section{Authors' contributions}

All authors contributed to developing the review protocol. LL conducted the literature searches, screening, and data extraction. DAJ conducted the initial $3 \%$ of the abstract screen to ensure reliability of the screening process. MS contributed to the study selection against inclusion/exclusion criteria. LL and MS wrote the first draft. The authors discussed, reviewed, and edited the manuscript and have approved the final version.

\section{Funding}

This work was funded by UKRI via the Alan Turing Institute under the Health and Medical Sciences Programme. DAJ is funded by the National Institute for Health Research Greater Manchester Patient Safety Translational Research Centre (NIHR Greater Manchester PSTRC). The views expressed are those of the author(s) and not necessarily those of the NHS, the NIHR, or the Department of Health and Social Care.

\section{Availability of data and materials}

Data sharing is not applicable to this article as no datasets were generated or analysed during the current study.

Ethics approval and consent to participate Not applicable.
Consent for publication

Not applicable.

\section{Competing interests}

The authors declare that they have no conflicts of interest relating to the publication of this work.

\section{Author details}

${ }^{1}$ Division of Informatics, Imaging and Data Science, Faculty of Biology, Medicine and Health, University of Manchester, Manchester Academic Health Science Centre, Manchester, UK. ${ }^{2}$ NIHR Greater Manchester Patient Safety Translational Research Centre, The University of Manchester, Manchester, UK. ${ }^{3} \mathrm{NIHR}$ Manchester Biomedical Research Centre, The University of Manchester, Manchester Academic Health Science Centre, Manchester, UK.

Received: 21 July 2020 Accepted: 2 January 202

Published online: 04 February 2021

\section{References}

1. Steyerberg EW. Clinical prediction models : a practical approach to development, validation, and updating: Springer; 2009. p. 497.

2. Hippisley-Cox J, Coupland C, Vinogradova Y, Robson J, May M, Brindle P. Derivation and validation of QRISK, a new cardiovascular disease risk score for the United Kingdom: prospective open cohort study. BMJ. 2007;335(7611):136 Available from: http://www.ncbi.nlm.nih.gov/pubmed/17615182.

3. Hippisley-Cox J, Coupland C, Brindle P. Development and validation of QRIS $\mathrm{K} 3$ risk prediction algorithms to estimate future risk of cardiovascular disease: prospective cohort study. BMJ. 2017;357:j2099 Available from: https://www.bmj.com/content/357/bmj.j2099.

4. NICE. Lipid modification: cardiovascular risk assessment and the modification of blood lipids for the primary and secondary prevention of cardiovascular disease. 2014. Available from: https://www.nice.org.uk/ guidance/cg181

5. van Geloven N, Swanson S, Ramspek C, Luijken K, Van Diepen M, Morris T, et al. Prediction meets causal inference: the role of treatment in clinical prediction models. Eur J Epidemiol. 2020; Available from: https://doi.org/10. 1007/s10654-020-00636-1.

6. Groenwold RHH, Moons KGM, Pajouheshnia R, Altman DG, Collins GS, Debray TPA, et al. Explicit inclusion of treatment in prognostic modeling was recommended in observational and randomized settings. J Clin Epidemiol. 2016;78:90-100 Available from: http://www.ncbi.nlm.nih.gov/ pubmed/27045189.

7. Liew SM, Doust J, Glasziou P. Cardiovascular risk scores do not account for the effect of treatment: a review. Heart. 2011;97(9):689-97. Available from: https://heart.bmj.com/content/97/9/689.

8. Pajouheshnia R, Schuster NA, Groenwold RH, Rutten FH, Moons KG, Peelen LM. Accounting for time-dependent treatment use when developing a prognostic model from observational data: A review of methods. Statistica Neerlandica. 2020;74(1):38-51. Available from: https://onlinelibrary.wiley. com/doi/abs/10.1111/stan.12193.

9. Lawton M, Tilling K, Robertson N, Tremlett H, Zhu F, Harding K, Oger J, BenShlomo Y. A longitudinal model for disease progression was developed and applied to multiple sclerosis. Journal of clinical epidemiology. 2015;68(11): 1355-65. Available from: https://doi.org/10.1016/j.jclinepi.2015.05.003.

10. Hernán MA, Hsu J, Healy B. A second chance to get causal inference right: a classification of data science tasks. CHANCE. 2019:32(1):42-9 Available from: https://www.tandfonline.com/doi/full/10.1080/09332480.2019.1579578.

11. Westreich D, Greenland S. The table 2 fallacy: presenting and interpreting confounder and modifier coefficients. Am J Epidemiol. 2013;177(4):292-8 Available from: http://www.ncbi.nlm.nih.gov/pubmed/23371353.

12. Shmueli G. To Explain or to Predict? Stat Sci. 2010;25(3):289-310 Available from: https://projecteuclid.org/euclid.ss/1294167961.

13. Dickerman BA, Hernán MA. Counterfactual prediction is not only for causal inference. Eur J Epidemiol. 2020;4 Available from: http://link.springer.com/1 0.1007/s10654-020-00659-8.

14. Arnold KF, Davies V, de Kamps M, Tennant PWG, Mbotwa J, Gilthorpe MS. Reflections on modern methods: generalized linear models for prognosis and intervention-theory, practice and implications for machine learning. Int J Epidemiol. 2020; Available from: https://pubmed.ncbi.nlm.nih.gov/323 80551/. 
15. Blakely T, Lynch J, Simons K, Bentley R, Rose S. Reflection on modern methods: when worlds collide-prediction, machine learning and causal inference. Int J Epidemiol. 2020;49(1):338-347. Available from: https://doi. org/10.1093/ije/dyz132.

16. Piccininni M, Konigorski S, Rohmann $J$, Kurth T. Directed acyclic graphs and causal thinking in clinical risk prediction modeling. BMC Med Res Methodol. 2020;20(1):179 Available from: https://bmcmedresmethodol.biomedcentral. com/articles/10.1186/s12874-020-01058-z.

17. Arksey H, O'Malley L. Scoping studies: towards a methodological framework. Int J Soc Res Methodol. 2005;8(1):19-32. Available from: https://doi.org/10. 1080/1364557032000119616.

18. Moher D, Shamseer L, Clarke M, Ghersi D, Liberati A, Petticrew M, et al. Preferred reporting items for systematic review and meta-analysis protocols (PRISMA-P) 2015 statement. Syst Rev. 2015;4(1) Available from: https:// systematicreviewsjournal.biomedcentral.com/articles/10.1186/2046-4053-4-1.

19. Martin GP, Jenkins D, Bull L, Sisk R, Lin L, Hulme W, et al. Towards a framework for the design, implementation and reporting of methodology scoping reviews. 2020;127:191. Available from: https://doi.org/10.1016/j. jclinepi.2020.07.014.

20. Geersing GJ, Bouwmeester W, Zuithoff P, Spijker R, Leeflang M, Moons K. Search filters for finding prognostic and diagnostic prediction studies in medline to enhance systematic reviews. PLoS One. 2012;7(2):e32844 Available from: http://www.ncbi.nlm.nih.gov/pubmed/22393453.

21. Nguyen T-L, Collins GS, Landais P, Le Manach Y. Counterfactual clinical prediction models could help to infer individualised treatment effects in randomised controlled trials - an illustration with the international stroke trial. J Clin Epidemiol. 2020;125:47-56 Available from: https://pubmed.ncbi. nlm.nih.gov/32464321/.

22. Candido dos Reis FJ, Wishart GC, Dicks EM, Greenberg D, Rashbass J, Schmidt MK, et al. An updated PREDICT breast cancer prognostication and treatment benefit prediction model with independent validation. Breast Cancer Res. 2017;19(1):58 Available from: http://breast-cancer-research. biomedcentral.com/articles/10.1186/s13058-017-0852-3.

23. Brunner FJ, Waldeyer C, Ojeda F, Salomaa V, Kee F, Sans S, et al. Application of non-HDL cholesterol for population-based cardiovascular risk stratification: results from the Multinational Cardiovascular Risk Consortium. Lancet. 2019;394(10215):2173-83 Available from: https://www.thelancet. com/journals/lancet/article/PIIS0140-6736(19)32519-X/fulltext.

24. Silva R. Observational-interventional priors for dose-response learning. in advances in neural information processing systems 29 (NIPS 2016); 2016. Available from: http://papers.neurips.cc/paper/6107-observationalinterventional-priors-for-dose-response-learning

25. van Amsterdam WAC, Verhoeff JJC, de Jong PA, Leiner T, MJC E. Eliminating biasing signals in lung cancer images for prognosis predictions with deep learning. NPJ Digit Med. 2019;2(1):1-6 Available from: https://www.nature. com/articles/s41746-019-0194-X.

26. Alaa AM, van der Schaar M. Bayesian inference of individualized treatment effects using multi-task gaussian processes. In: Advances in Neural Information Processing Systems 30 (NIPS 2017); 2017. Available from: https://papers.nips.cc/paper/6934-bayesian-inference-of-individualizedtreatment-effects-using-multi-task-gaussian-processes.

27. Arjas E. Time to consider time, and time to predict? Stat Biosci. 2014;6(2): 189-203 Available from: https://link.springer.com/article/10.1007/s12561013-9101-1.

28. Sperrin M, Martin GP, Pate A, Van Staa T, Peek N, Buchan I. Using marginal structural models to adjust for treatment drop-in when developing clinical prediction models. Stat Med. 2018;37(28):4142-54 Available from: http://doi. wiley.com/10.1002/sim.7913.

29. Lim B. Forecasting treatment responses over time using recurrent marginal structural networks. In: 32nd Conference on Neural Information Processing Systems (NeurIPS 2018); 2018. p. 7494-504. Available from: http://papers nips.cc/paper/7977-forecasting-treatment-responses-over-time-usingrecurrent-marginal-structural-networks.

30. Bica I, Alaa AM, Jordon J, van der Schaar M. Estimating counterfactual treatment outcomes over time through adversarially balanced representations. In: 8th International Conference on Learning Representations (ICLR); 2020. Available from: https://openreview.net/pdf?id $=\mathrm{BJg} 866 \mathrm{NFvB}$.

31. $X u Y, X u Y$, Saria S. A Bayesian nonparametric approach for estimating individualized treatment-response curves. In: Doshi-Velez F, Fackler J, Kale D, Wallace B, Wiens J, editors. Proceedings of the 1st Machine Learning for
Healthcare: PMLR; 2016. p. 282-300. Available from: http://proceedings.mlr. press/v56/Xu16.pdf.

32. Soleimani H, Subbaswamy A, Saria S. Treatment-response models for counterfactual reasoning with continuous-time, continuous-valued interventions. In: the 33rd Conference on Uncertainty in Artificial Intelligence (UAI); 2017. Available from: http://auai.org/uai2017/proceedings/ papers/266.pdf.

33. Schulam P, Saria S. Reliable decision support using counterfactual Models. In: Advances in Neural Information Processing Systems 30 (NIPS 2017); 2017. p. 1697-708. Available from: https://papers.nips.cc/paper/6767-reliabledecision-support-using-counterfactual-models.

34. Pearl J. Causality: Models, reasoning, and inference, second edition: Cambridge University Press; 2011. p. 1-464.

35. Li J, Zhao L, Tian L, Cai T, Claggett B, Callegaro A, et al. A predictive enrichment procedure to identify potential responders to a new therapy for randomized, comparative controlled clinical studies. Biometrics. 2016;72(3): 877-87 Available from: https://pubmed.ncbi.nlm.nih.gov/26689167/.

36. Lamont A, Lyons MD, Jaki T, Stuart E, Feaster DJ, Tharmaratnam K, et al. Identification of predicted individual treatment effects in randomized clinical trials. Stat Methods Med Res. 2018;27(1):142-57 Available from: https://pubmed.ncbi.nlm.nih.gov/26988928/.

37. Cai T, Tian L, Wong PH, Wei LJ. Analysis of randomized comparative clinical trial data for personalized treatment selections. Biostatistics. 2016;17(2):24963 Available from: https://www.lanternpharma.com/.

38. Daley DJ, Vere-Jones D. An introduction to the theory of point processes: Volume I: Elementary, Theory and Methods - Second Edition. Probability and its Applications. 2003.

39. Rasmussen CE, CKI W. Gaussian processes for machine learning: the MIT Press; 2006. Available from: www.GaussianProcess.org/gpml

40. Robins J. Association, causation, and marginal structural models. Synthese 1999;121(1/2):151-79.

41. Farmer RE, Kounali D, Walker AS, Savović J, Richards A, May MT, et al. Application of causal inference methods in the analyses of randomised controlled trials: a systematic review. Trials. 2018;19(1):23 Available from: https://trialsjournal.biomedcentral.com/articles/10.1186/s13063-017-2381-x.

42. Glymour C, Zhang K, Spirtes P. Review of causal discovery methods based on graphical models. Front Genet. 2019;10(JUN):524 Available from: https:// www.frontiersin.org/article/10.3389/fgene.2019.00524/full.

43. Tennant PW, Harrison WJ, Murray EJ, Arnold KF, Berrie L, Fox MP, et al. Use of directed acyclic graphs (DAGs) in applied health research: review and recommendations. medRxiv. 2019; Available from: https://www.medrxiv.org/ content/10.1101/2019.12.20.19015511v1.

44. Clare PJ, Dobbins TA, Mattick RP. Causal models adjusting for time-varying confounding — a systematic review of the literature. Int J Epidemiol. 2019;(1):48, 254-265 Available from: http://www.ncbi.nlm.nih.gov/pubmed/30358847.

45. Frank Harrell, Laura Lazzeroni. EHRs and RCTs: outcome prediction vs. optimal treatment selection [Internet]. 2020 [accessed 2020 Apr 19]. Available from: https://www.fharrell.com/post/ehrs-rcts/

46. Kent DM, Paulus JK, van Klaveren D, D'Agostino R, Goodman S, Hayward R, et al. The Predictive Approaches to Treatment effect Heterogeneity (PATH) Statement. Ann Intern Med. 2020;172(1):35 Available from: https://annals. org/aim/fullarticle/2755582/predictive-approaches-treatment-effectheterogeneity-path-statement.

47. Kent DM, van Klaveren D, Paulus JK, D'Agostino R, Goodman S, Hayward R, et al. The Predictive Approaches to Treatment effect Heterogeneity (PATH) statement: explanation and elaboration. Ann Intern Med. 2020;172(1):W1-25 Available from: https://www.acpjournals.org/doi/10.7326/M18-3668.

48. Bica I, Alaa AM, Lambert C, van der Schaar M. From real-world patient data to individualized treatment effects using machine learning: current and future methods to address underlying challenges. Clin Pharmacol Ther. 2020;cpt:1907 Available from: https://onlinelibrary.wiley.com/doi/abs/10.1 002/cpt.1907.

49. Jain P, Danaei G, Manson JE, Robins JM, Hernán MA. Weight gain after smoking cessation and lifestyle strategies to reduce it. Epidemiology. 2020; 31(1):7-14 Available from: http://journals.Iww.com/00001648-202001000 00002.

50. Lusivika-Nzinga C, Selinger-Leneman H, Grabar S, Costagliola D, Carrat F. Performance of the marginal structural cox model for estimating individual and joined effects of treatments given in combination. BMC Med Res Methodol. 2017:17(1):1-11 Available from: https://bmcmedresmethodol. biomedcentral.com/articles/10.1186/s12874-017-0434-1. 
51. Vangen-Lønne AM, Ueda P, Gulayin P, Wilsgaard T, Mathiesen EB, Danaei G. Hypothetical interventions to prevent stroke: an application of the parametric g-formula to a healthy middle-aged population. Eur J Epidemiol. 2018;33(6):557-66.

52. Orellana L, Rotnitzky A, Robins J. Generalized marginal structural models for estimating optimal treatment regimes; 2006.

53. van der Laan MJ, Petersen ML. Causal effect models for realistic individualized treatment and intention to treat rules. Int J Biostat. 2007;3(1): Article3 Available from: https:/www.ncbi.nlm.nih.gov/pmc/articles/ PMC2613338\%

54. Robins JM. Optimal structural nested models for optimal sequential decisions. In: Lin DY, Heagerty PJ, editors. Proceedings of the Second Seattle Symposium in Biostatistics. New York, NY: Springer; 2004. p. 189-326.

55. Chakraborty B, Murphy SA. Dynamic treatment regimes. Annu Rev Stat Its Appl. 2014;1 (1):447-64 Available from: http://www.annualreviews.org/doi/10 1146/annurev-statistics-022513-115553.

56. Pajouheshnia R, Peelen LM, Moons KG, Reitsma JB, Groenwold RH. Accounting for treatment use when validating a prognostic model: a simulation study. BMC Med Res Methodol. 2017;17(1):103. Available from: https://doi.org/10.1186/s12874-017-0375-8

57. Alaa $A M$, van der Schaar M. Validating causal inference models via influence functions. In: 36th International Conference on Machine Learning, ICML 2019; 2019. p. 281-91. Available from: http://proceedings.mlr.press/v97/alaa1 9a.html.

58. Franks AM, D'Amour A, Feller A. Flexible sensitivity analysis for observational studies without observable implications. J Am Stat Assoc. 2020:115:(532): 1730-46. Available from: https://doi.org/10.1080/01621459.2019.1604369.

\section{Publisher's Note}

Springer Nature remains neutral with regard to jurisdictional claims in published maps and institutional affiliations.

Ready to submit your research? Choose BMC and benefit from:

- fast, convenient online submission

- thorough peer review by experienced researchers in your field

- rapid publication on acceptance

- support for research data, including large and complex data types

- gold Open Access which fosters wider collaboration and increased citations

- maximum visibility for your research: over $100 \mathrm{M}$ website views per year

At $\mathrm{BMC}$, research is always in progress.

Learn more biomedcentral.com/submission 